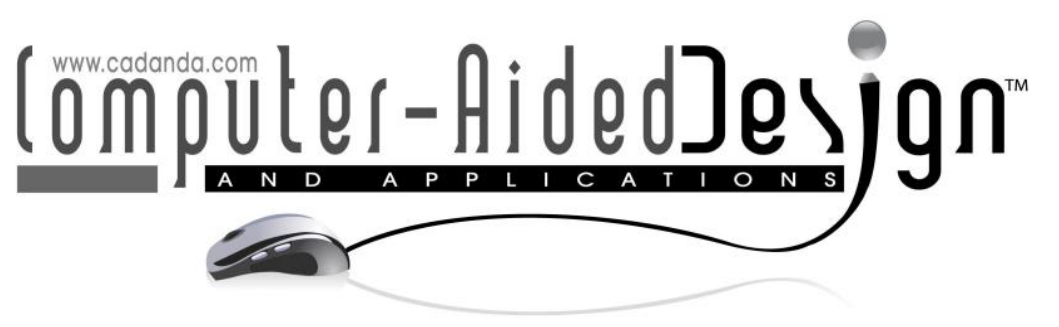

\title{
Rigid 3D Registration Algorithm for Localization of the Vertebral Centroids in 3D Deformity Models of Adolescent Idiopathic Scoliosis
}

\author{
Saša Ćukovići (D), William Taylor $^{2}$ (D), Vanja Luković3 (D), Ionut Ghionea ${ }^{4}$ (D), Khelifa Baizid ${ }^{5}$ (D), \\ Jamshed Iqbal ${ }^{6}$ (D) and Subburaj Karuppasamy ${ }^{7}$ (D) \\ ${ }^{1}$ University of Kragujevac, Faculty of Engineering, Serbia, cukovic@kg.ac.rs \\ ${ }^{2}$ Swiss Federal Institute of Technology, ETH Zurich, Switzerland, bt@ethz.ch \\ 3University of Kragujevac, Faculty of Technical Sciences, Serbia, vanja.lukovic@ftn.kg.ac.rs \\ ${ }^{4}$ University Politehnica of Bucharest, Romania, ionut.ghionea@imst.pub.ro \\ ${ }^{5}$ Istituto Italiano di Tecnologia, Genova, Italy, khelifa.baizid@iit.it \\ 6University of Jeddah, Saudi Arabia, iqbal.jam@gmail.com \\ ${ }^{7}$ Singapore University of Technology and Design, Singapore, subburaj@sutd.edu.sg
}

Corresponding author: Saša M. Ćuković, cukovic@kg.ac.rs

\begin{abstract}
In this paper, we developed a methodology for a non-invasive 3D diagnosis based on a digitized dorsal surface of the patient enabling assessment and monitoring of Adolescent Idiopathic Scoliosis (AIS). We applied a novel 3D registration algorithm, which enables rigid transformation of 3D vertebral CAD models and localization of vertebrae centroids on the middle 3D spinal line to simulate the AIS. Registrations of the vertebral centroids on a 5th degree middle spinal B-Spline curve is the main step in establishing biomechanical relations of internal structure (deformed spine) with a digitized surface of patients and extracting the key diagnostic parameters of the AIS. This simulation algorithm is developed into PLM system CATIA using KAx tools and successfully evaluated on 372 AIS patients.
\end{abstract}

Keywords: Scoliosis, 3D Spine, Rigid 3D Registration, AIS Modeling

DOI: https://doi.org/10.14733/cadaps.2020.1313-1325

\section{INTRODUCTION}

Scoliosis is a highly complex 3D musculoskeletal disorder (MSD) of the spine that greatly affects the patient's health and quality of life, including functional limitations with secondary complications such as respiratory and circulatory dysfunction, pain, and cosmetic changes. Scoliosis affects $2-4 \%$ of the global population, and almost $80 \%$ of the developed cases are of an unknown reason (i.e. idiopathic) [6], with an estimated prevalence of 36 million new patients worldwide by 2050 according to the WHO. Adolescent idiopathic scoliosis (AIS) is defined as a deformation of the 
spinal curve of more than $10^{\circ}$ angle measured traditionally on standing planar or biplanar radiographs, using the Cobb technique [5].

To-date, the so-called Cobb angle is considered as the key parameter in AIS diagnosis and acts as a guide for treatment, but describes only the 2D curvature of the deformity in the anterior/posterior and/or sagittal plane from 2D X-ray images with significant intra- and interobserver variability and errors [24].

The main drawback of traditional diagnostic methods in AIS assessment is their harmful effect on this vulnerable population, especially, due to multiple exposures to radiation (e.g. X-rays, EOS, $\mathrm{CT}$ ) during the monitoring process. As a result, due to the repetitive test images, patients are exposed to a substantial amount of ionizing radiation [14], and each exposure increases the risk of cancer development. However, it is mandatory for patients with AIS to be examined every 3-6 months to monitor their curvature's progression and treatment outcomes [22]. Although new technologies have significantly reduced radiation intensity (e.g. EOS), there is still an increased cumulative risk for patients [17]. Extensive research [25] in 5573 female breast cancer patients showed that $69 \%$ had some exposure to ionizing techniques for spinal deformity diagnosis during adolescence. The clinical goal is, therefore, to avoid or reduce application of those methods in deformity monitoring [15] with a preference for non-invasive techniques based on visual examination of the patient's trunk and external deformity measures [11],[14], but this is also highly subjective and based upon the clinician's experience.

In recent years, innovative 3D optical systems have been considered as alternatives to X-ray imaging for estimating the 3D spinal curve that passes through centroids of each vertebral body (Middle Spinal Line - MSL) and vertebral axial rotations [2],[16]. A surface topography method, e.g. rasterstereography, is less expensive, non-invasive and non-ionizing technique through which the curvature of the spine is modelled to allow an estimation and evaluation of deformity-related changes of the digitized patient's back surface using only light as a medium [26]. New studies have shown that certain deformity indicators (Cobb angle) calculated using optical methods have a relatively high reliability [23],[29]. However, to-date, none of these diagnostic approaches have been applied to calculate other internal parameters of the deformity, and still neglect the 3D complexity of the internal spinal structures.

A generic, state-of-the-art 3D CAD model of the spine (GMS) has been recently developed at the University of Kragujevac to simulate the 3D nature of the deformity and to estimate internal biomechanical properties and indicators of AIS. GMS is scalable and model of the spine that can be registered to the 3D digitized surfaces of the patient's back, optically (non-invasively) scanned [6]. Also, a novel computational algorithm embedded in the state-of-the-art ScolioSIM1.0 tool for markerless and contactless detection of the key anatomical landmarks on a patient's back was developed and tested on partial X-Ray images [6]. These technical solutions resolve our challenge to generate a 3D patient-specific spine/deformity (PSS) model and to extract internal deformity parameters from an external surface only.

In addition to the 3D nature of spinal deformities, the degree of progression as the child matures is the fourth dimension of AIS, adding further challenges to the clinical assessment and management. Namely, the majority of AIS patients experience progression of their curvature after reaching skeletal maturity and the risk of progression in adulthood and associated health problems increases significantly [4],[10],[33]. Accordingly, monitoring of AIS is essential to recognize the changes at an early stage and counteract with a therapy, as with a spinal curvature of more than $50^{\circ}$ surgical treatment is recommended to reduce the curve, stop the progression and maintain lung and heart function.

\section{NEW NON-INVASIVE 3D DIAGNOSIS}

We proposed a non-invasive 3D methodology and a system to quantify deformity measures using patient-specific models generated from patient's dorsal shape, anatomical landmarks, the curve of surface asymmetry and middle spinal curve generated from optical 3D scan data. Developed 
system is created using knowledgeware technology and VBA macros implemented in PLM system CATIA V5 to perform these measurements with minimal human intervention. A general methodology for obtaining a patient-specific 3D model of the deformity is given in Figure 1.

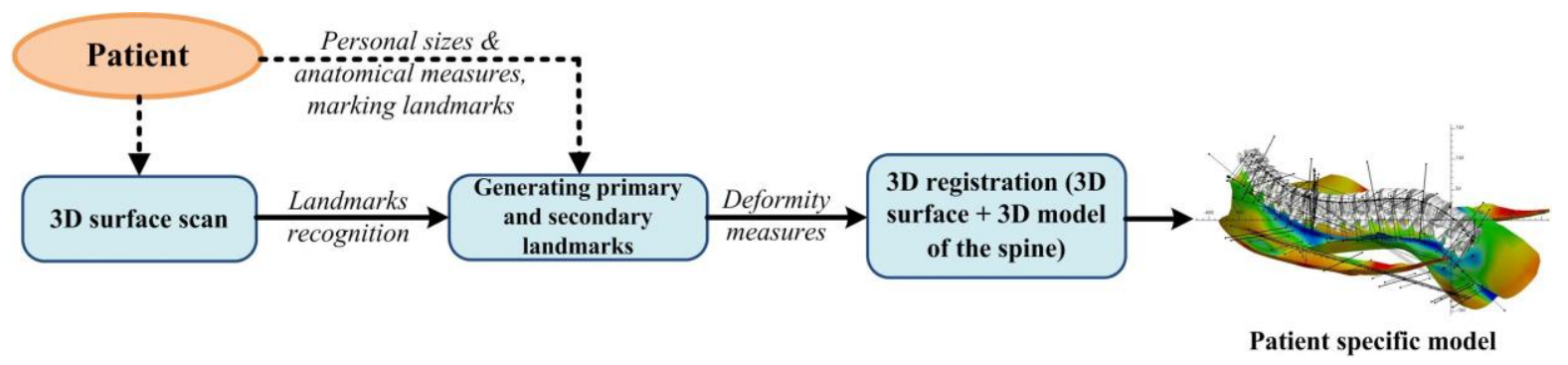

Figure 1: Stages of generating a 3D model of the deformity - patient specific model.

\subsection{Materials and Methods}

In this research, to simulate patient-specific model of spinal deformity and its evaluation we used the following datasets, materials and methods:

- Patient datasets (372 patients);

- 3D scanning and reconstruction of the dorsal surface using ARTEC Eva device or equivalent;

- 3D spinal reconstruction - Generic 3D spinal model using Materialise Mimics software;

- Surface importing and analysis in PLM system CATIA and curvature analysis of the dorsal surface;

- Application of Knowledgeware technologies and scripts:

- extracting dorsal asymmetry line, middle spinal line and curvature analysis of those lines in 3 planes - 3D analysis of the spinal deformity;

- creating reference elements for vertebral models' orientation towards the dorsal surfaces;

- mathematical measurements of deformity indicators (internal and external);

- 3D visualization of the deformity model, apical vertebrae, primary and secondary curve detection.

- Reports on 3D analysis and visualizations and statistical analysis.

\subsection{Knowledgeware Technologies}

We used PLM system of CATIA V5R20 to automate the methodology with an in-built VBA (VB software is given in [20]) scripts called ScoliosisSimulator-3DSpinalRegistration.catvba. This macro processes the patient's optical scan of the dorsal surface and generates elements of CAD skeletal model based on a generic parameterized 3D model of spine (by rigid registration) and generates key parameters to quantify deformity [6],[8].

We implemented VBA macro for generating and visualizing reference elements of the 3D skeletal model on the spinal curve. For this purpose, we used Turner-Smith's rule [6], which localize peaks of spinal processes towards the dorsal surface. The VBA macro produces a set of diagnostic parameters exported in a separate *.xls file for further analysis.

\subsubsection{D registration of the spinal model with the dorsal surface - "Patient-specific" 3D deformity model}

Generic 3D model of the spine is adaptable and it can be registered to the dorsal surface. Using ScoliosisSimulator-3DSpinalRegistration.catvba macro, "patient-specific" model of deformity is generated. The scaling factor will modify each vertebral model in three directions according to rigid registration principle (3D to 3D), Figure 2. 


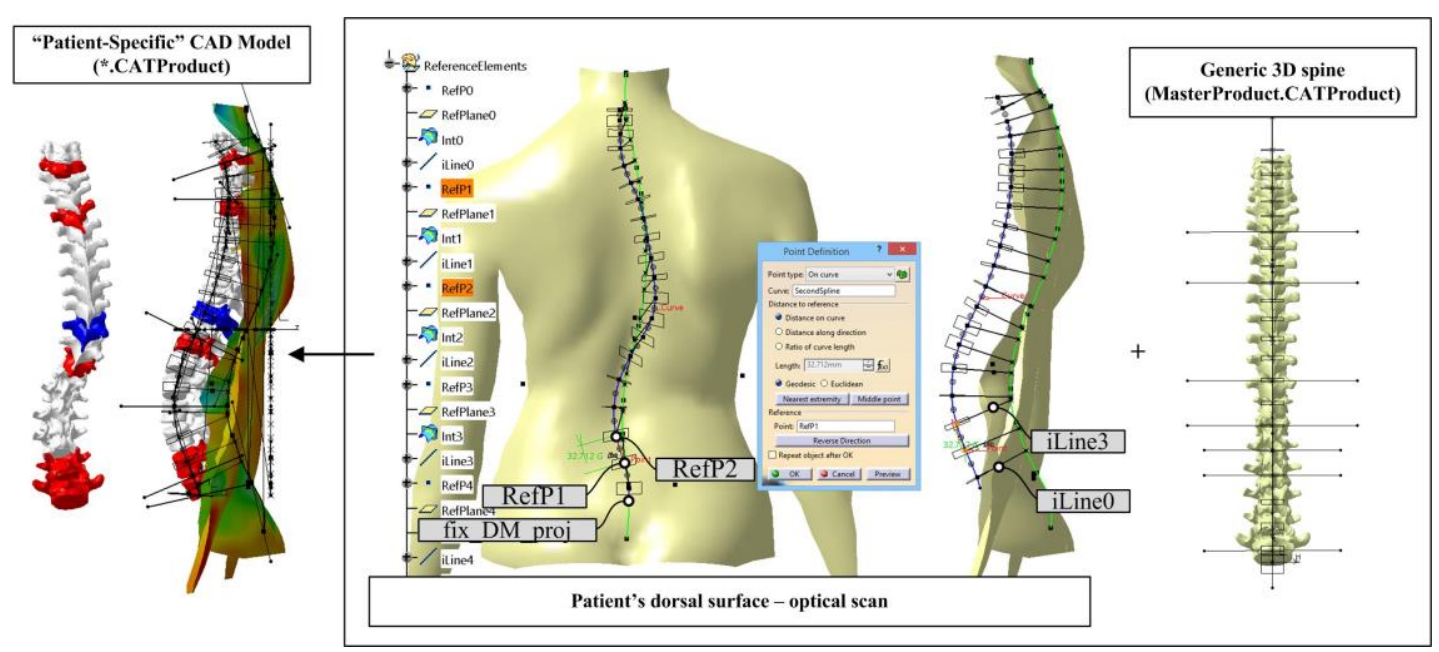

Figure 2: Registration and referencing of the generic 3D model of the spine to the dorsal surface "patient-specific" model of the deformity.

This model generates a set of internal and external parameters. Particularly important are: SOSORT-these angles, Cobb angles, transpositions of vertebral body centroids and intervertebral discs [5],[6],[14]. Transpositions have negative sign ("-") if the vertebral bodies are dislocated left from CVS (Central Vertical Spinal) line, and positive ("+") if centroids are right from this line. The vertebra which is in a sense of absolute value, the mostly dislocated, is called apical vertebra. This vertebra belongs to the primary curve and is around of the peak that curve [27].

The "gold standard" in radiographic measurement of spinal curve and its progression in clinical practice over time is a Cobb angle in frontal and sagittal planes, Figure 3 [7]. This angle quantifies a curvature of the curve between end vertebras of the deformity curve in frontal and sagittal planes, and angles greater than 10 degrees are considered as a significant for scoliosis diagnosis.

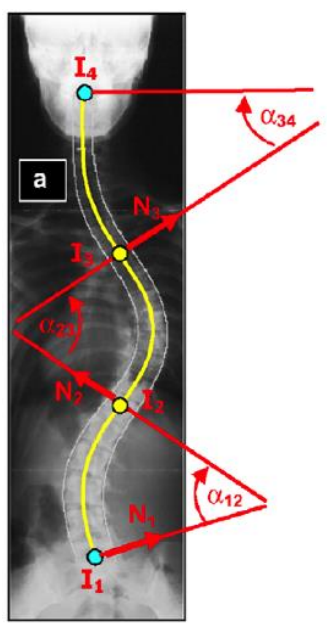

a)
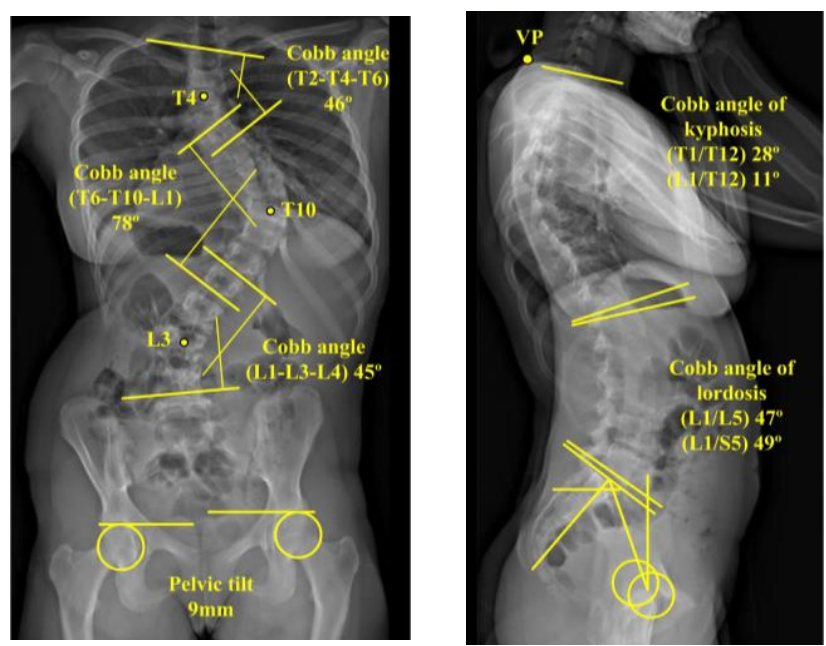

b)

Figure 3: Cobb angle measurement on X-ray films a) triple curve with Cobb angles $\alpha_{12}, \alpha_{23}, \alpha_{34}, \mathrm{~b}$ ) illustration of manual measurements of Cobb angles in frontal and sagittal planes. 
SOSORT consortium recommends selecting the reference vertebrae in the sagittal and frontal planes for measuring specific angles as it is done in the clinical settings [14]. Thus, we included SOSORT-these angles between vertebrae T2-T5, T5-T12, T10-L2, and L1-L5 in the frontal plane and angle between vertebrae L1-L5 and T4-T12 in the sagittal plane.

To localize centroids of each vertebra and transform 3D vertebral models to make them "patient-specific" we implemented registration technique. Vertebral models are then registered over the skeletal model of the deformity, Figure 4.

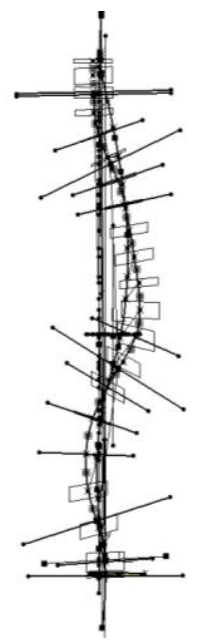

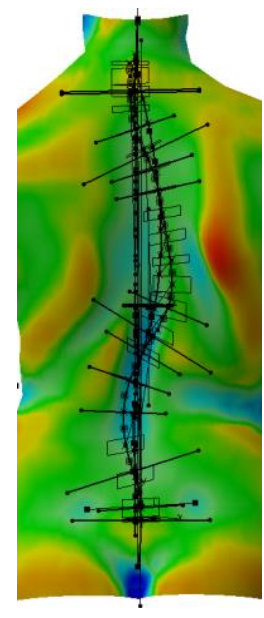

a)

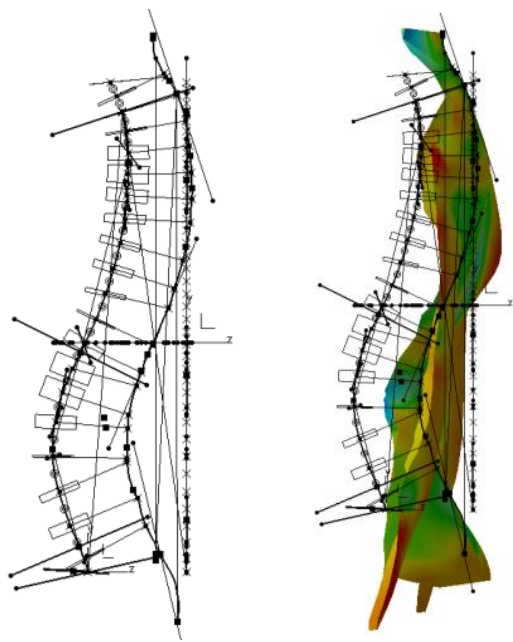

b)

Figure 4: Reference skeletal model of the AIS deformity on a) frontal and b) sagittal plane.

\section{REGISTRATION}

Image Registration is a set of transformation methods used to fuse two 2D images or 3D point clouds in a common coordinate system. It is usually considered that raw images or 3D clouds of the target object are acquired in different time and perspective, with the same (intramodal) or different modalities (intermodal) [1].

Registration algorithms determine corresponding elements of the two images (fixed and floating) and calculate geometrical transformation for their spatial alignment (term registration or alignment can be found in literature as Matching, Stabilization, Fusion, Stitching, etc.). The first type of registration was rigid and includes: scaling, rotation and translation of the correspondent features or landmarks of the flowing image to a fixed image. Deformable registration is a set of non-rigid geometrical transformations used for fusion of highly complex deformable shapes or images.

Deformable registration is applicable in soft tissue reconstruction and functional analysis (lung function, brain cancer monitoring, prostate cancer diagnosis, heart-rate image compensation, etc.). In some cases, it is necessary to combine rigid registration as initialisation of the fusion process and to complete registration with some non-rigid algorithm. There are many registration algorithms applicable in medicine available today $[13],[18],[19],[28]$.

\subsection{D/3D Registration}

In the case of 3D-3D registration, it is necessary to have corresponding points, lines or surface features, internal or external anatomical landmarks. Vertebral models of the generic spine are reconstructed from CT slices and represent point clouds of the real vertebrae [9]. We used CATIA to make scalable and parametric models adaptable to various patient-specific conditions, including 
congenital changes on vertebral shapes (Figure 5). These models are statistical and correspond to standardized Panjabi's measurements [6].

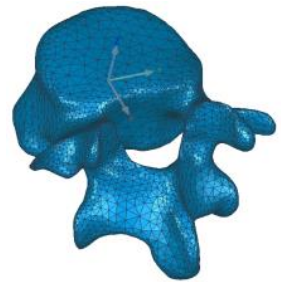

a)

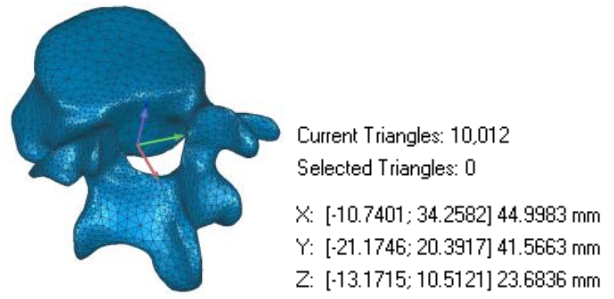

b)

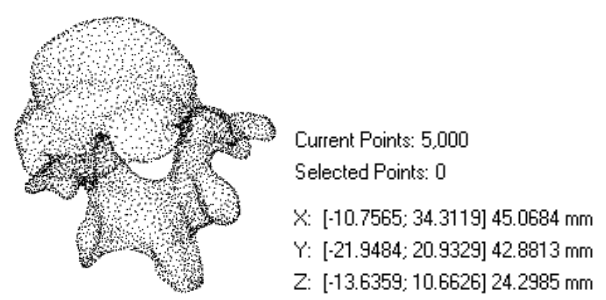

c)

Figure 5: Statistical/generic model of L4 vertebra a) original, b) translated in its centroid, c) point cloud.

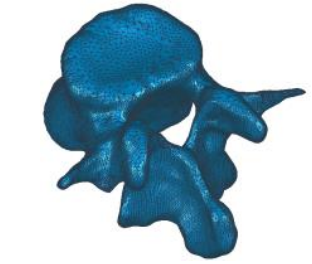

Current Triangles: 38,362

Selected Triangles: 0

X: $[-41.0737 ; 41.0737] 82.1474 \mathrm{~mm}$

$Y:[-38.2838 ; 38.2838] 76.5676 \mathrm{~mm}$

Z: $[-21.4517 ; 21.4517] 42.9034 \mathrm{~mm}$

a)

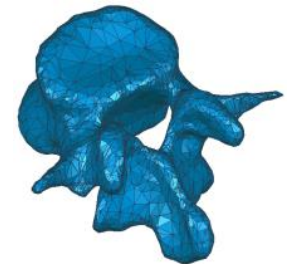

Current Triangles: 5,754

Selected Triangles: 0

$X:[-106.7721 ;-24.5521] 82.2200 \mathrm{~mm}$

$Y:[-38.3445 ; 38.4043] 76.7489 \mathrm{~mm}$

Z: $[33.1521 ; 76.0128] 42.8607 \mathrm{~mm}$

b)

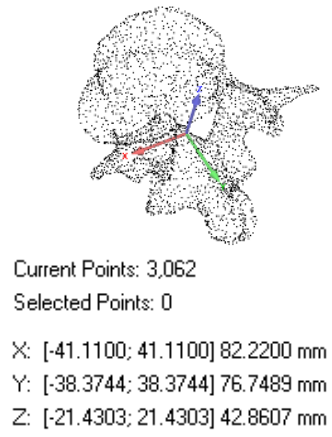

C)

Figure 6: Targeted model of L4 vertebra a) polygonal, b) 15\% decimated, c) point cloud.

In some cases when registration shapes are very complex it is not always convenient to detect corresponding elements between them. In this scenario, it is better to find centroids of point cloud's volume and then proceed with registration [31]. One of the recently developed registration algorithms is CPD-Coherent Point Drift is particularly interesting as it combines rigid and deformable transformations and perfectly fits for vertebral shapes, and it is resistant to a different number of floating cloud (Figure 5) and a targeted shape (Figure 6).

In this paper, we apply a 3D registration algorithm to transform vertebral models and adopt their positions toward 3D back surface. As we work with bone anatomical 3D models, we focused on rigid $3 \mathrm{D}$ transformation algorithms.

\subsection{D Transformations: Rotation, Translation, Scaling}

Vertebral models are 3D representations of real vertebrae (hard structure) reconstructed over CT images, scalable and parametrically described. To register their centroids on the middle spinal line and orient them towards 3D back surface we applied 3D transformations (rotation, translation and scaling) that are necessary to describe registration function given in equation $4.1[8],[12]$.

\subsection{General Methodology}

We consider the alignment of two vertebral shapes (point clouds) as a probability density estimation problem defined by Myronenko. One of the point clouds is GMM (Gaussian mixture model) centroids and another one is the target point cloud [3]. Bellow, we give a basic notation, as it is defined in [21]: 
- $\quad D$ - dimension of the point sets; $N, M$ - number of points in the point sets;

- $X_{N \times D}=x_{1}, \ldots x_{N}{ }^{T}$ - the first point set; $Y_{M \times D}=y_{1}, \ldots y_{M}{ }^{T}$ - the second point set (GMM centroid);

- $\tau=Y, \theta$ - transformation $\tau$ applied to $Y$, where $\theta$ is a set of the transformation parameters;

- 1 - column vector of all ones;

- $\quad d=(a)$ - diagonal matrix formed from vector $a$.

Rigid registration allows rigid transformations: translation, rotation and uniform scaling factor $[21],[30]$.

\section{RIGID REGISTRATION}

For this type of registration, the transformation of the GMM is required $\tau y_{m} ; R, t, s=s R y_{m}+t$ where $R_{D \times D}$ is a rotation matrix, $t_{D \times 1}$ is a translation vector and $s$ is a scaling factor. In this case, the objective $Q$ - function depends on rotation, translation and scaling factor and has the form (4.1):

$$
\begin{gathered}
Q R, t, s, \sigma^{2}=\frac{1}{2 \sigma^{2}} \sum_{n=1}^{N} \sum_{m=1}^{N} P^{\text {old }}\left(m \mid x_{n}\right)\left\|x_{n}-s R y_{m}-t\right\|^{2}+\frac{N_{P} D}{2} \log \sigma^{2} \\
\text { Where: } R^{T} R=I \text { and } \operatorname{det}(R)=1
\end{gathered}
$$

In the given equation, the first part is similar to form of absolute orientation problem [32].

\subsection{Application of Rigid Registration to AIS Modelling}

\subsubsection{D spinal assembly model}

To simulate scoliosis of a specific patient, it is necessary to have a fully assembled model of the physiologically normal spine with 20 vertebral models. This CAD model is a parametric master model created in CATIA and represents the " $\mathrm{S}$ " shaped spine with normal lumbar lordosis, thoracic kyphosis and cervical lordosis (Figure 7).
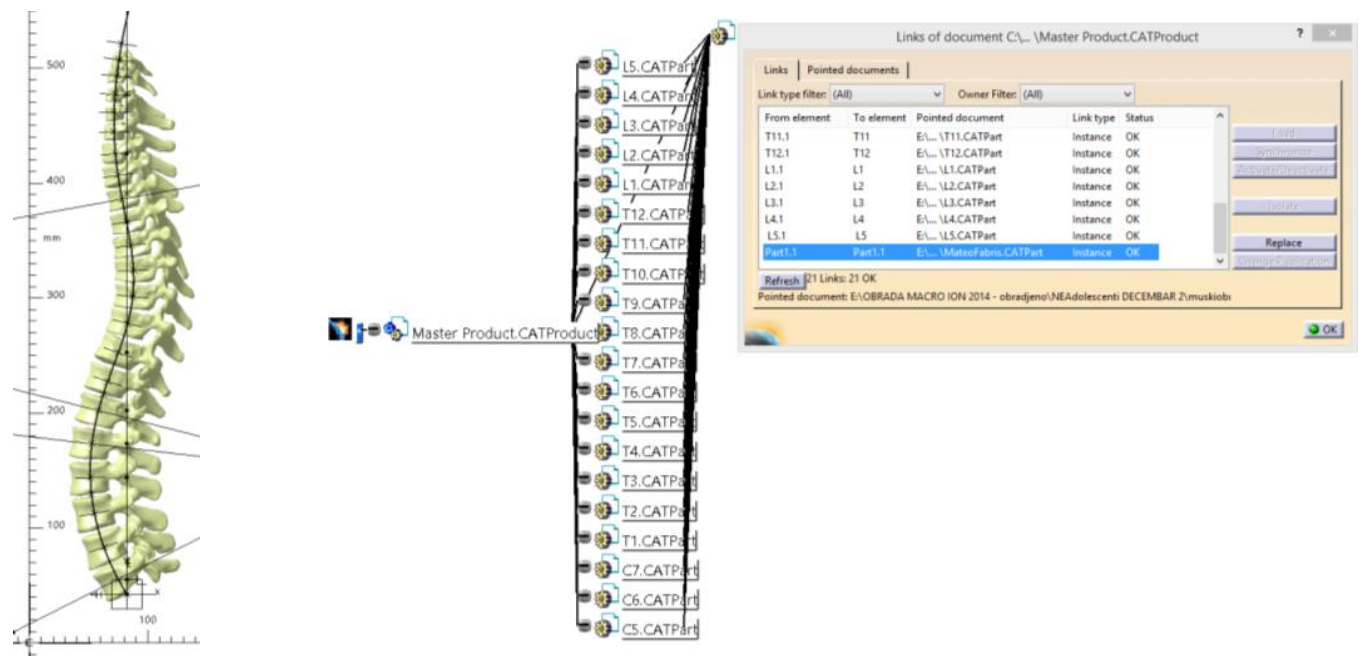

Figure 7: 3D CAD model of a generic spine (Master Product.CATProduct) - Physiologically normal spine. 
After many tests in clinical environment we concluded that 5 th degree B-Spline curve is the best fitting solution for AIS representation on X-Ray films and for approximation of the real spinal curve, to calculate inflection points and to set reference lines for measuring Cobb angles in two planes [7]. In this course, we used 5th degree B-Spline curve to localise vertebral centroids on it.

\subsubsection{Referencing of the vertebral models to dorsal surfaces}

Master 3D model of the spine is adoptable to 3D dorsal skeletal models generated using ScoliosisSimulator-3DSpinalRegistration.catvba macro illustrated in Figure 4 . This is done by rigid transformations using embedded formula (4.1) over common referencing elements of the skeletal model and vertebra (centroid and vertebral axes). Figure 8 a illustrates referencing and orientation of the lumbar model L2 towards digitized dorsal surface and automatic creation of the geometrical relations among geometrical elements in assembly model (e.g. coincidence).

Orientation of each vertebral model is defined according to the Turner-Smith's rule. This rule defines the coincidence between vertebral vector $\vec{n}$ which passes through vertebral centroid and procesus spinosus and normal vector of the back surface $\vec{n}_{s}$, Figure $8 \mathrm{~b}$. This rule is also important to calculate parameters of axial vertebral rotation of each vertebra in deformity models.

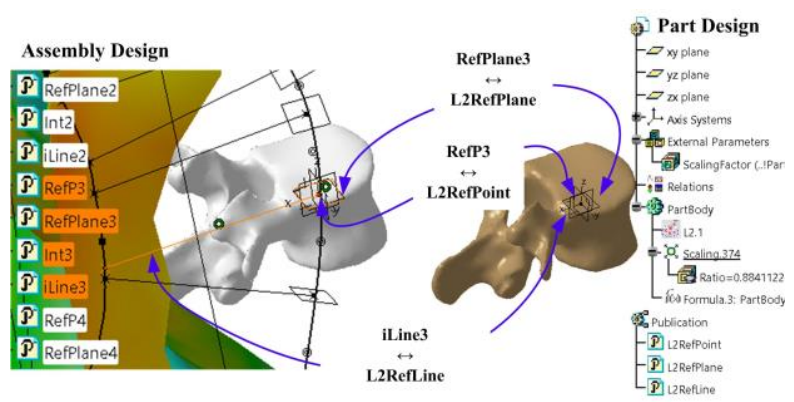

a)

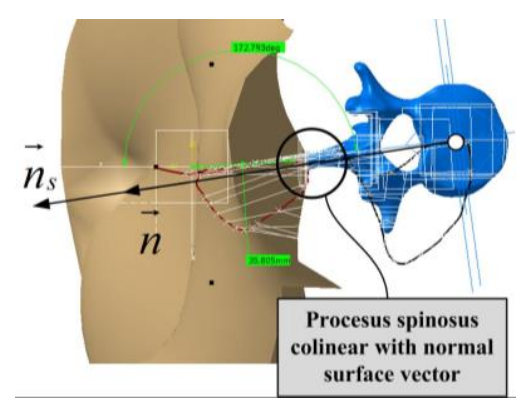

b)

Figure 8: Referencing of the lumbar model L2 towards back surface a) over reference elements, and b) Turner-Smith's rule.

VBA script also creates all dependencies between skeletal deformity model of the dorsal surface and enables strong multi-modular associations and built-in knowledge with vertebral models from the master assembly (Master Product.CATProduct). Initialization of the registration process is the determination of the scaling factor and its transfer from skeletal deformity model to each vertebra model, as it is illustrated in Figure 9.

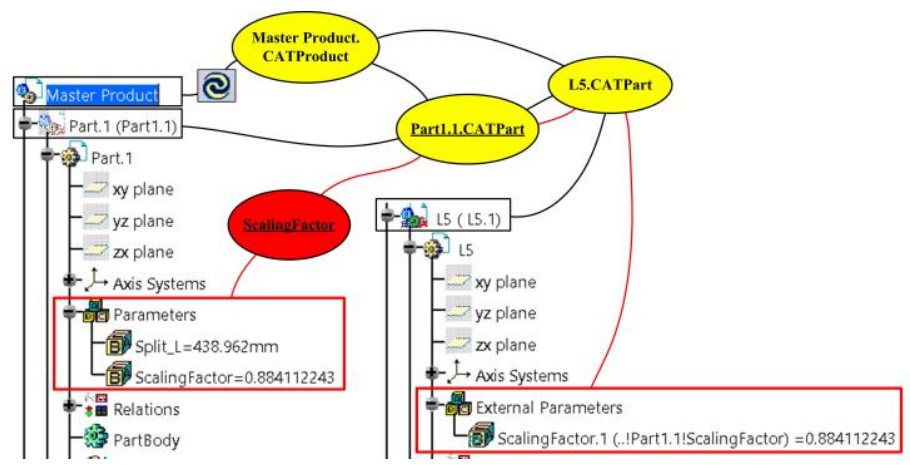

Figure 9: Initialisation of the rigid registration process and regenerating vertebral models to patient's back surface. 
Standardized modelling techniques, like publication, "child-parent" modelling technique as well as external parametrization in assembly module, also, are implemented. After registering all vertebral models towards surfaces, we have a full 3D deformity model of AIS, or "patient-specific" deformity model (Figure 10).

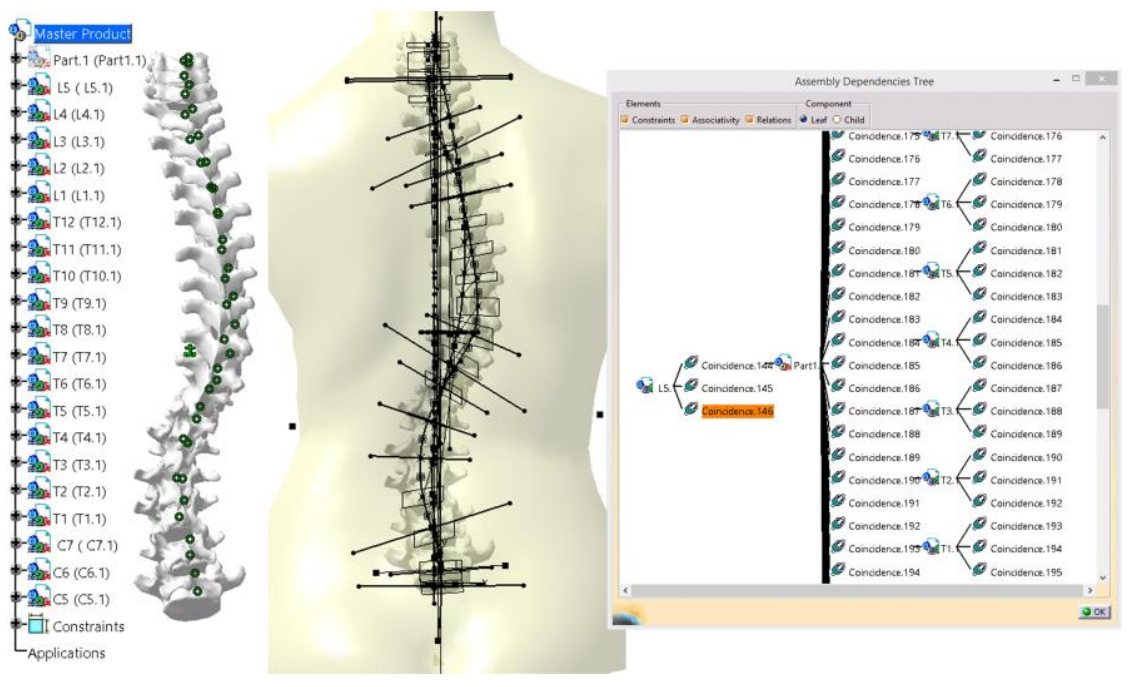

Figure 10: Example of AIS patient-specific assembly model in CATIA with model tree and geometrical associations after the registration process for a 14 years old female patient.

Once the patient-specific model is generated, the initial generic model (Part1.1.CATPart) will be replaced with the patient-specific model in the data flow. 3D CAD model of deformity and ScoliosisSimulator-3DSpinalRegistration.catvba macro are integral part of the system ScolioMedIS, the information system for monitoring and diagnosing scoliosis, for online AIS diagnosis and monitoring.

\section{RESULTS}

The system produced a set of diagnostic parameters that are displayed in the special branch of the CAD models tree, but also exported in separate *.xls file for downstream analysis. Besides, it generates a printable report on the performed analysis (Figure 11).

Full visualization of the deformity is performed using Knowledgeware technologies and rigid registration algorithm in a standard $C A D$ environment. As results, of the registration process and mathematical calculation we have many parameters generated in the sub-processes. This includes:

- Automatic detection of anatomical landmarks on the patient's back surface;

- Generated asymmetry line of the dorsal surface and middle spinal line as a $5^{\text {th }}$ degree BSpline curve;

- Reference elements for dorsal deformity indicators measurements;

- Key initial parameters of the "Patient-Specific" model (Scaling Factor, Split_L, etc.);

- Reference elements for 3D rigid registration of vertebral models, positioning, translation, scaling and rotation;

- Inflection points of the middle spinal line in frontal and sagittal planes and corresponding Cobb angles;

- Detected apex vertebrae and end vertebrae of the primary and secondary curves of the deformity greater than $10^{\circ}$ according to Cobb;

- Transpositions of the vertebral models from specific vertical axes of the patient; 
- $\quad$ SOSORT-these angles [6],[14];

- 3D visualiyations of the deformity in 3D with ready for archiving and online visualization (*.xIs, *.CATPart, *.CATProduct, *.STL, *.txt, *.3DXml), etc.

We successfully tested abovementioned system on 372 patients in adolescence optically diagnosed from 2015 to 2017. Following patient cases are examples of two female patients with moderate thoracic scoliosis:

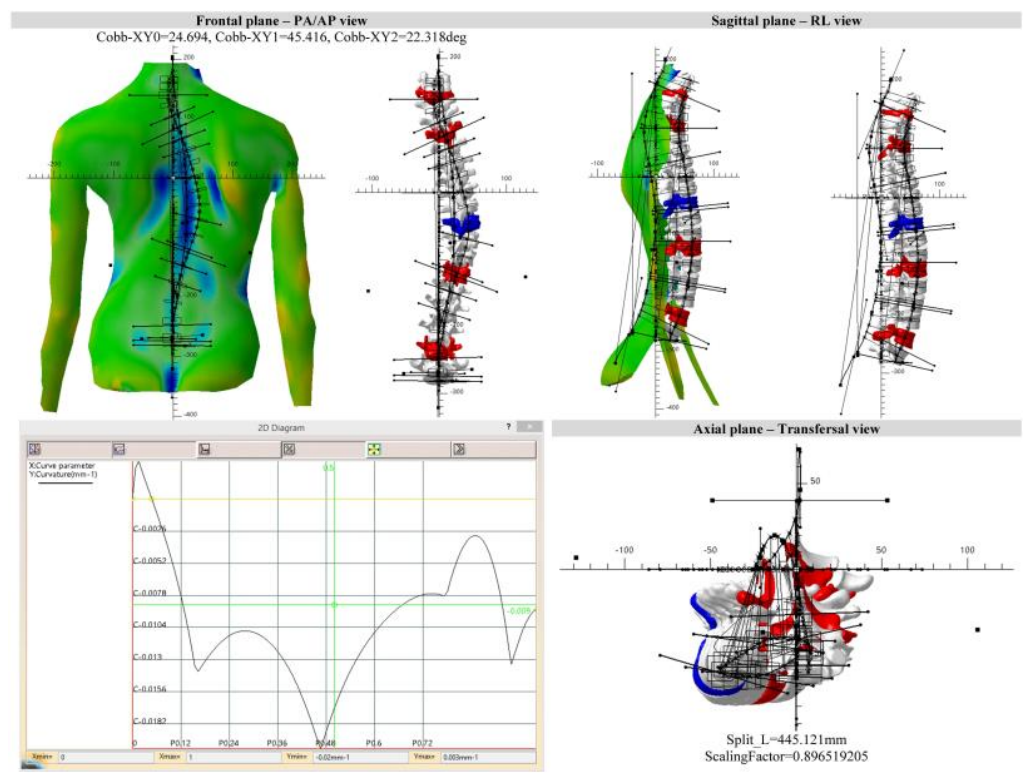

a)

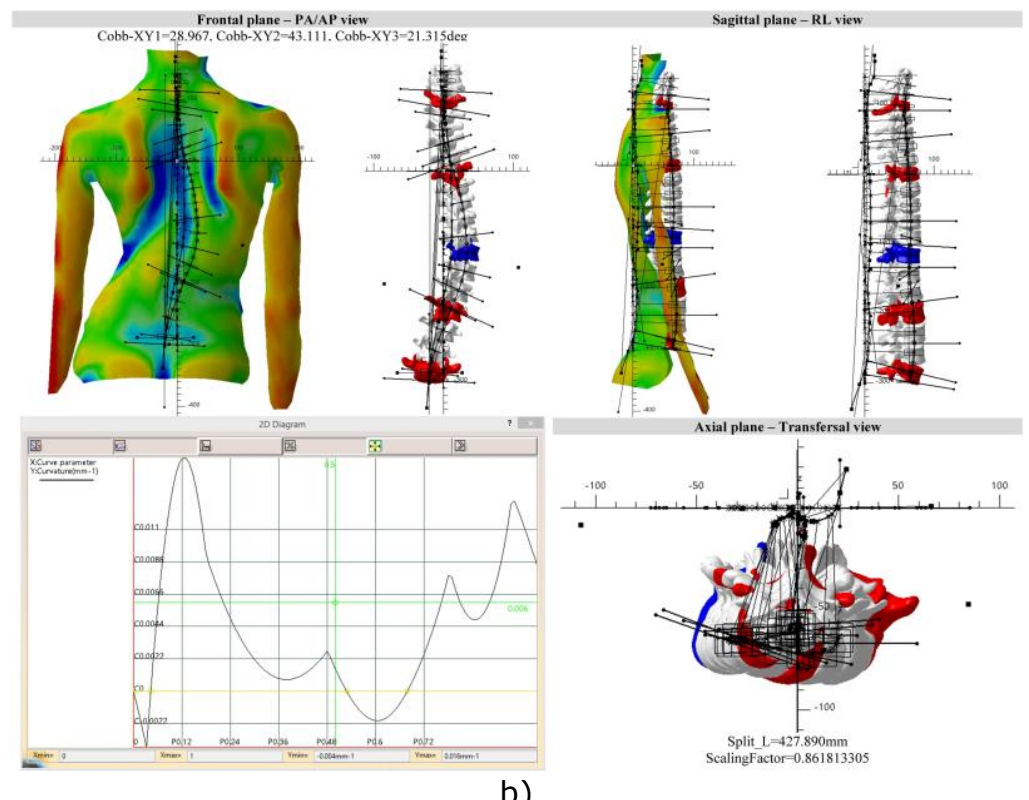

Figure 11: Reports and visualizations of the Patient-specific deformity models for two AIS patients. 


\section{CONCLUSIONS AND FUTURE WORK}

This paper presents a part of the results of the development of the methodology for the diagnosis of spinal deformities. It is based on the application of methods of surface topography and reduced exposure to X-rays for patients suffering from idiopathic progressive scoliosis, aimed at elimination of radiation. The selected approach is based on the application of knowledgeware technology, as the integrator for patient-specific 3D modelling, visualization, simulation and monitoring of scoliosis. Spinal deformity modelling is based on a generic 3D model of the spine, generated from CT scans, which can be regenerated and adapted to the model of the patient's dorsal surface. The developed system is tested on a representative number of adolescent (372), using models of their dorsal surfaces, results indicate that the system is robust and it can reduce the need for radiographic examinations.

Although the incidence and prevalence of scoliosis are very high, there still is not a unique protocol for its clinical assessment and monitoring. The protocol, we proposed takes into consideration the 3D nature of the deformity, with a special accent on the sagittal plane and axial rotations, which is usually given insufficient attention in everyday clinical practice. Our system excludes subjective assessment and any writing/reading error, and it can be expected that this protocol will gain high value when measuring on subjects with scoliosis are performed. As the system is based on precise, quantitative and objective procedure for the analysis and visualization of human static posture on the basis of the detected anatomical landmarks, it can give its potential applications and further development directions.

We presented, in this paper, a 3D rigid registration algorithm to orient and register CAD vertebral models toward digitally reconstructed back surface of patients. This process is the key to get patient-specific model of deformity and to generate a very important set of extrinsic and extrinsic deformity indicators in diagnosis and monitoring process. Further efforts will be focused on the identification of typical morphological characteristics of deformities that would enable 3D deformable registration to adopt the algorithm with the congenital spinal deformities, grouping and comparing deformity lines and improve diagnosis process and precision of deformity models.

Current version of the system is tested at Center for Physical Medicine and Rehabilitation, in Orthopedics and Traumatology Clinic in Kragujevac, Serbia, and its implementation showed promising results, particularly in adolescent idiopathic deformities.

Saša Ćuković, https://orcid.org/0000-0003-0525-2197

William Taylor, https://orcid.org/0000-0003-4060-4098

Vanja Luković, http://orcid.org/0000-0002-1887-6102

Ionut Ghionea, http://orcid.org/0000-0002-9062-0064

Khelifa Baizid, https://orcid.org/0000-0002-3438-9291

Jamshed Iqbal, https://orcid.org/0000-0002-0795-0282

Subburaj Karuppasamy, https://orcid.org/0000-0003-0723-2405

\section{REFERENCES}

[1] Ardeshir, G.: 2-D and 3-D Image Registration for Medical, Remote Sensing, and Industrial Applications, John Wiley \& Sons, Inc., 2005.

[2] Aroeira, R. M.; Leal, J. S.; de Melo Pertence, A. E.: New method of scoliosis assessment: Preliminary results using computerized photogrammetry, Spine, 36(19), 2011, 1584-1591. https://doi.org/10.1097/BRS.0b013e3181f7cfaa

[3] Bing, J.; Baba, C. V.: Robust point set registration using Gaussian mixture models, IEEE Transactions on Pattern Analysis and Machine Intelligence, 33(8), 2011, 1633-1645. https://doi.org/10.1109/TPAMI.2010.223

[4] Cheung, J. P. Y.; Cheung, P. W. H.; Samartzis, D.; Luk, K. D.: Curve progression in adolescent idiopathic scoliosis does not match skeletal growth, Clinical Orthopaedics and Related Research, 476(2), 2018, 429-436. https://doi.org/10.1007/s11999.0000000000000027 
[5] Cobb, J. R.: Outline for the Study of Scoliosis. In: Instructional Course Lectures. The American Academy of Orthopaedics Surgeons, 1948, 261-275.

[6] Ćuković, S.: Non-rigid Registration of Sculptured Surfaces in Internet Environment, PhD Thesis, UniKg, Faculty of Engineering, Kragujevac, Serbia, 2015.

[7] Ćuković, S; Devedžić, G.; Luković, V.; Anwer, A.; Zečević-Luković, T.; Karupppasamy, S.: 3D Modeling of spinal deformities shapes using 5th degree B-splines, Journal of Production Engineering, 18(2), 2015, 103-106.

[8] Devedžić, G.; Ćuković, S.; Luković, V.; Milošević, D.; Subburaj, K.; Luković, T.: ScolioMedIS: Web-oriented information system for idiopathic scoliosis visualization and monitoring, Computer Methods and Programs in Biomedicine, 108(2), 2012, 736-749. https://doi.org/10.1016/j.cmpb.2012.04.008

[9] Devedzic, G.; Ristic, B.; Stefanovic, M.; Cukovic, S.; Lukovic, T.: Development of 3D parametric model of human spine and simulator for biomedical engineering education and scoliosis screening, Journal of Computer Applications in Engineering Education, 20(3), 2010, 434-444. https://doi.org/10.1002/cae.20411

[10] Di Felice, F.; Zaina, F.; Donzelli, S.; Negrini, S.: The natural history of idiopathic scoliosis during growth: A meta-analysis, American Journal of Physical Medicine \& Rehabilitation, 97(5), 2018, 346-356. https://doi.org/10.1097/PHM.0000000000000861

[11] Drerup, B.: Rasterstereographic measurement of scoliotic deformity, Scoliosis, 9(22), 2014, 1-18. https://doi.org/10.1186/s13013-014-0022-7

[12] Haidekker, M.: Advanced Biomedical Image Analysis, Singapore: John Wiley \& Sons, Inc., Hoboken, New Jersey, 2011.

[13] Joseph, V. H.; Derek, L. G. H.; David, J. H.: Medical Image Registration, Biomedical Engineering Series ed., Boca Raton, Florida: CRC Press LLC, 2001.

[14] Knott, P.; Eden, P.; Michelle, C.; JC deMauroy; Charles, R.; Tomasz, K.; Fabio, Z.; James, W.; Luke, S.; Josette, B. S.; Theodoros, B. G.; Jacek, D.; Toru, M.; Stefano, N.; Joseph, P. O'B.; Manuel, R.: SOSORT 2012 Consensus Paper: Reducing x-ray exposure in pediatric patients with scoliosis, Scoliosis, 2014; 9(4). https://doi.org/10.1186/1748-7161-9-4

[15] Komeili, A.; Westover, L. M.; Parent, E. C.; Moreau, M.; El-Rich, M.; Adeeb, S.: Surface topography asymmetry maps categorizing external deformity in scoliosis, Spine, 14(6), 2014, 973-983. https://doi.org/10.1016/j.spinee.2013.09.032

[16] Lam, G. C.; Hill, D. L.; Le, L. H.; Raso, J. V.; Lou, E. H.: Vertebral rotation measurement: A summary and comparison of common radiographic and CT methods, Scoliosis, 3(16), 2018, 1-10. https://dx.doi.org/10.1186\%2F1748-7161-3-16

[17] Law, M.; Ma, W. K.; Lau, D.; Cheung, K.; Ip, J.; Yip, L.; Lam, W.: Cumulative effective dose and cancer risk for pediatric population in repetitive full spine follow-up imaging: How micro dose is the EOS microdose protocol?, European Journal of Radiology, 101, 2018, 87-91. https://doi.org/10.1016/j.ejrad.2018.02.015

[18] Markelj, P.; Tomazevic D.; Likar, B.; Pernus, F.: A review of 3D/2D registration methods for image-guided interventions, Journal of Medical Image Analysis, 16(3), 2012, 642-661. https://doi.org/10.1016/j.media.2010.03.005

[19] Medha, V. W.; Pradeep, M. P.; Hemant, K. A.: Image registration techniques: An overview, International Journal of Signal Processing, Image Processing and Pattern Recognition, 2(3), 209, 11-28.

[20] Microsoft, December 2018. [Online]. Available: http://www.microsoft.com/enus/download/confirmation.aspx?id=10019

[21] Myronenko, A.; Xubo, S.: Point set registration: Coherent point drift, IEEE Transactions on Pattern Analysis and Machine Intelligence, 32(12), 2010, 2262-2275. https://doi.org/10.1109/TPAMI.2010.46

[22] Patias, P.; Grivas, T. B.; Kaspiris, A.; Aggouris, C.; Drakoutos, E.: A review of the trunk surface metrics used as scoliosis and other deformities evaluation indices, Scoliosis, 5(12), 2010, 1-20. https://doi.org/10.1186/1748-7161-5-12 
[23] Rehm, J.; Thomas, G.; Michael, A.; Wojciech, P.; Hans-Ulrich, K.; Marc-André, W.; Daniel, S.: 3D-modeling of the spine using EOS imaging system: Inter-reader reproducibility and reliability, PLoS One, 12(2), 2017, e0171258. https://dx.doi.org/10.1371\%2Fjournal.pone.0171258

[24] Richards, B. S.; Sucato, D. J.; Konigsberg, D. E.; Ouellet, J. A.: Comparison of reliability between the Lenke and King classification systems for adolescent idiopathic scoliosis using radiographs that were not premeasured, Spine, 28(11), 2003, 1148-1157. https://doi.org/10.1097/01.BRS.0000067265.52473.C3

[25] Ronckers, C. M.; Land, C. E.; Miller, J. S.; Stovall, M.; Lonstein, J. E.; Doody, M. M.: Cancer mortality among women frequently exposed to radiographic exams for spinal disorders, Radiation Research, 174(1), 2010, 83-90. https://doi.org/10.1667/RR2022.1

[26] Schülein, S.; Mendoza, S.; Malzkorn, R.; Harms, J.; Skwara, A.: Rasterstereographic evaluation of interobserver and intraobserver reliability in postsurgical adolescent idiopathic scoliosis patients, Journal of Spinal Disorders \& Techniques, 26(4), 2013, E143-9. https://doi.org/10.1097/BSD.0b013e318281608c

[27] Stokes, I. A. F.: Three-dimensional terminology of spinal deformity. A report presented to the scoliosis research society by the scoliosis research society working group on $3 \mathrm{~d}$ terminology of spinal deformity, Spine, 19(2), 1994, 236-248.

[28] Suri, S. J.; David, L. W.; Swamy, L.: Handbook of Biomedical Image Analysis - Volume III: Registration Models, Kluwer Academic / Plenum Publishers, New York, 2005.

[29] Tabard-Fougère, A.; Bonnefoy-Mazure, A.; Hanquinet, S.; Lascombes. P.; Armand, S.; Dayer, R.: Validity and reliability of spine rasterstereography in patients with adolescent idiopathic scoliosis, Spine, 42(2), 98-105. https://doi.org/10.1097/BRS.0000000000001679

[30] Terry, S. Y.: Insight into Images: Principles and Practice for Segmentation, Registration, and Image Analysis, Weliesey: A K Peters, Ltd., 2004.

[31] Tomazevic, D.; Likar, B.; Slivnik, T.; Pernus, F.: 3-D/2-D registration of CT and MR to x-Ray images, IEEE Transactions on Medical Imaging, 22(11), 2003, 1407-1416. https://doi.org/10.1109/TMI.2003.819277

[32] Umeyama, S.: Last-squares estimation of transformation parameters between two-point patterns, IEEE Transactions on Pattern Analysis and Machine Intelligence, 13(4), 1991, 376380.

[33] Weinstein, S. L.; Ponseti, I. V.: Curve progression in idiopathic scoliosis, Journal of Bone and Joint Surgery, 65(4), 1983, 447-455. 